\title{
Nucleation phenomena in a multi-stage low pressure steam turbine
}

\author{
D E Bohn ${ }^{1}$, N Sürken ${ }^{1}$ and $\mathbf{F}$ Kreitmeier ${ }^{2}$ \\ ${ }^{1}$ Institute of Steam and Gas Turbines, Aachen University, Aachen, Germany \\ ${ }^{2}$ Alstom Power, Baden, Switzerland
}

\begin{abstract}
During the expansion of steam in the low pressure (LP) stages of steam turbines, the originating two-phase wet-steam mixture causes considerable thermodynamic losses as well as aerodynamic losses. The reduction of these loss mechanisms is the subject of research project at the Institute of Steam and Gas Turbines, Aachen University.

A three-dimensional nucleating wet steam flow with homogeneousheterogeneous nucleation in the three front stages of an industrial LP-steam turbine is investigated numerically. The steady, viscous, and compressible metastable steam flow calculations are performed with a Navier-Stokes flow solver incorporating the IAPWS-IF97 steam tables. A union numerical approach for both the homo- and heterogeneous nucleation occurring on soluble nuclei is employed to capture the effects related to the nucleation phenomenon. The model links the interfacial surface tension, the size of nuclei, the chemical characteristics of the substances forming the droplets, and the expansion rate with the nucleation rate. In order to take into account the additional viscous effects due to shrouded bladings, the open shroud cavities are modeled in detail. Droplet density spectra, radial droplet number, droplet diameter, and wetness fraction distributions at the exit of the third stage are calculated.

It is shown that impurities can cause nucleation to appear at lower supersaturations with higher nucleation rates compared to homogeneous nucleation of pure steam. In this way, thermodynamic and kinematic relaxation losses are reduced. Owing to the dissipative viscous effects near the endwalls, the nucleation fronts exhibit convex shapes. They are locally bound within the region of high expansion rates in the second stage's nozzle guide vane. For both heterogeneous and homogenous nucleating flows the wetness is highly dispersed with narrow droplet density spectra behind the three front stages.
\end{abstract}

Keywords: nucleation, two-phase flow, steam turbine, multi-stage calculation, wetness loss, IAPWS-IF97

\section{INTRODUCTION}

During the expansion of steam in turbines, after crossing the saturation line the fluid first supercools, its free energy reaches its maximum value and then initiates nucleation. Nucleated droplets continue to grow if their size is greater than the critical droplet size. This two-phase wet-steam mixture, consisting of a very large number of extremely small droplets, causes thermodynamic as well as aerodynamic losses in the low pressure (LP) stages of a steam turbine.

One of the first approaches to the homogeneous nucleation theory with respect to the condensing phase was suggested by Oswatitsch [1]. Later, Frenkel [2], Dunning

The MS was received on 31 March 2003 and was accepted after revision for publication on 9 May 2003.

*Corresponding author: Institute of Steam and Gas Turbines, Aachen University, Templergraben 55, 52056 Aachen, Germany.
[3] and McDonald [4] developed a theory of formation of critical droplets in a pure vapour.

Early investigations of homogeneous nucleating wetsteam flow have been performed in nozzles. Following the validation of theoretical analyses with experimental results, the theory of homogeneous condensation was also applied to LP-steam turbines. As a consequence, a number of investigations have been performed using various theories in order to analyse homogeneously nucleating steam flows in turbines [4-6].

The heterogeneous condensation phenomenon was first recognized at the beginning of the 19th century in the area of cloud microphysics. Köhler [7] was the first to derive a theoretical expression for the variation of vapour pressure over the curved surface of an aqueous solution drop. Fletcher [8] summarized a theoretical system for analysing the condensation phenomenon of water vapour in the atmosphere. 


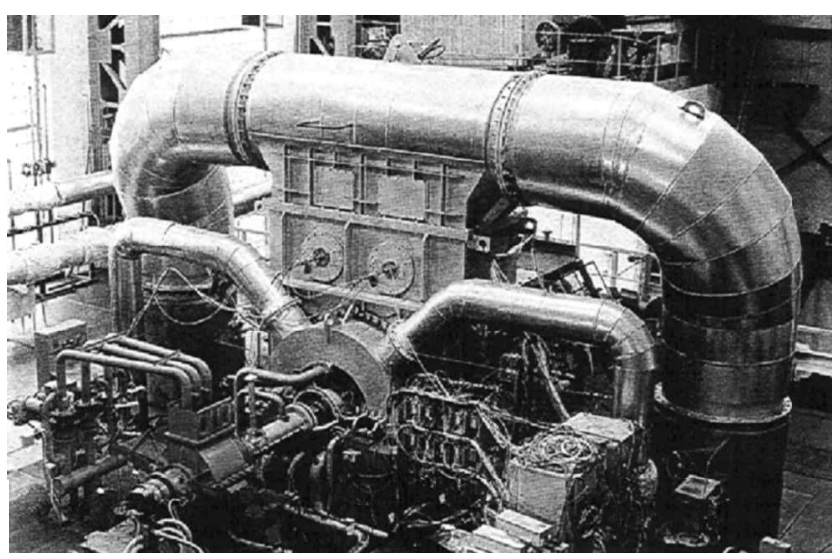

Fig. 1 Test facility (Source: Kreitmeier et al. [13])

Schnerr [9] dealt with heterogeneous nucleation in nozzles by reducing considerably the free energy barrier, which is a function of the contact angle, the radius of nuclei, and of particles. Stastny and Sejna [10] developed an approach to simulate the interacting heterogeneous and homogeneous nucleation in LP-steam turbines. Gorbunov [11] developed an approach to calculate the free energy of embryo formation for multicomponent heterogeneous nucleation. It is considered in his model that a liquid droplet is formed as a result of the deposition of several gaseous compounds onto a nucleus. Bohn and Ren [12] investigated heterogeneous nucleation in a two-dimensional nozzle guide vane by establishing a numerical nucleation model with consideration of chemical components.

Besides all this outstanding theoretical and basic work, strongly coupled close-to-industrial-application experimental and numerical work has also to be performed, in order to judge the importance of the different theoretical aspects for the nucleation phenomenon in real steam turbines. The present text contains results of an investigation of threedimensional nucleating wet steam flow with homogeneous/ heterogeneous nucleation in the three front stages of an industrial LP-steam turbine. Steady, viscous, and compressible metastable steam flow calculations are performed with an in-house Navier-Stokes flow solver incorporating the IAPWS-IF97 steam tables.

Figure 1 shows the test facility on which the numerical analyses are based. It is a 0.316 downscaled large LP-steam turbine with an axial/radial diffusor, three axial front stages with a cylindrical hub, and two final stages (Fig. 2, left) [13]. Control surfaces for flow field measurements are located before the three front stages (CS 20), behind the third stage (CS 42), behind the fourth (CS 52), and behind the final stage (CS 62). The operating conditions derived from the corresponding full-scale turbine are also shown in Fig. 2, right-hand side [13].

\section{MAIN SUBJECT MATTER}

\subsection{Nucleation model}

A union numerical approach for both the homo- and heterogeneous nucleation occurring on soluble nuclei is employed to capture effects related to the nucleation phenomenon. The model links the interfacial surface tension, the size of the nuclei, the chemical characteristics of the substances forming the droplets, as well as the expansion rate with the nucleation rate $[\mathbf{1 4}, \mathbf{1 5}]$.

Concerning a low concentration of an impurity (for example, $\mathrm{NaCl}$ ) in the steam, it is assumed that only supersaturated water vapour molecules condense on the soluble nuclei present during the expansion, and because Gibb's free energy and therefore the droplet nucleation rate are strongly sensitive to surface tension, the surface tension of the
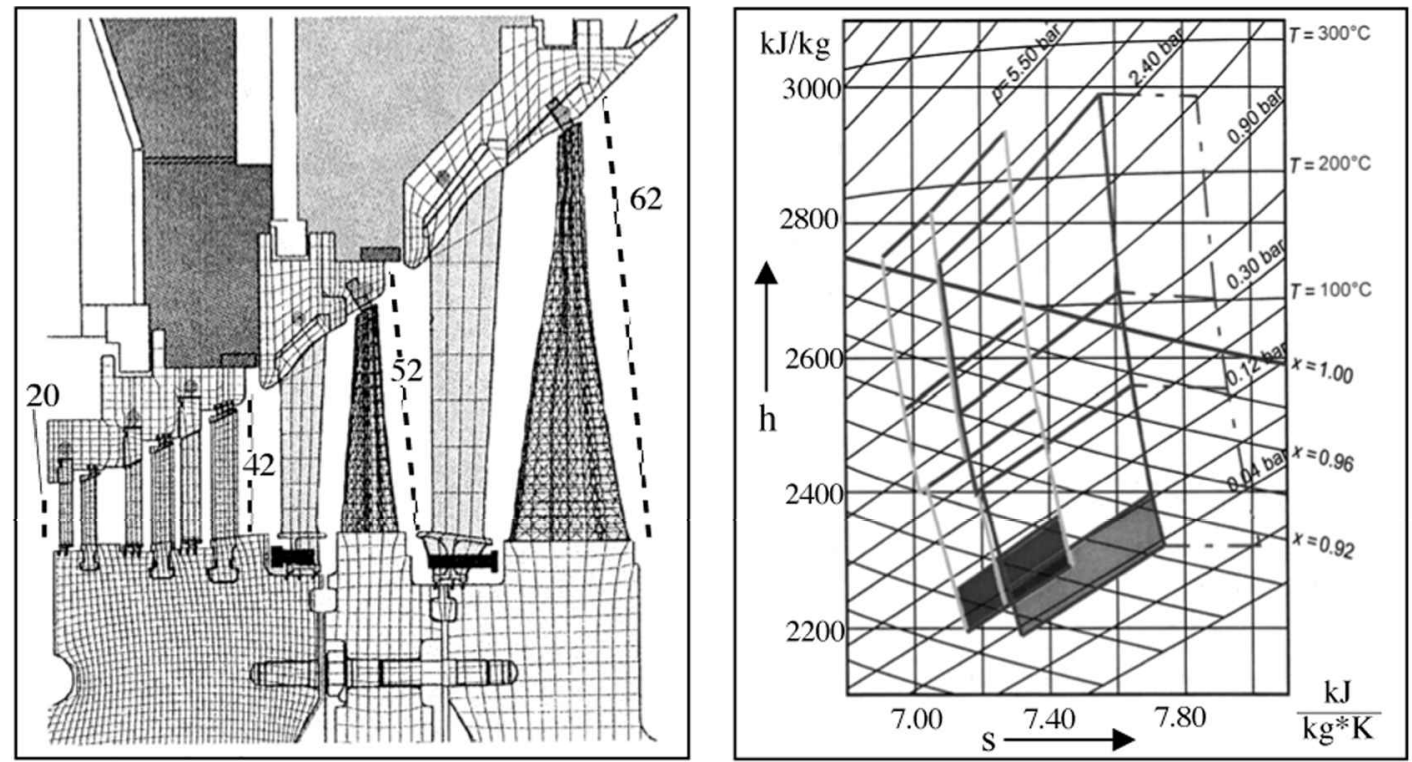

Fig. 2 Blading of the steam turbine with control surfaces and operating conditions (Source: Kreitmeier et al. [13]) 
chemical droplet $\sigma_{\mathrm{S} / \mathrm{G}}$ is calculated as a function of steam temperature $T_{\mathrm{G}}$, salt solution (molality), and radius of the chemical droplet.

The maximum free energy $\Delta G^{*}$ and the corresponding nucleation rate of critical chemical droplets per unit volume $J$ are then calculated by:

$$
\begin{aligned}
& \Delta G^{*}=4 \pi r^{* 2} \sigma_{\mathrm{S} / \mathrm{G}}+\frac{4}{3} \pi r^{* 3} \rho_{\mathrm{L}} R_{\mathrm{G}} T_{\mathrm{G}} \ln \left(\frac{a_{\mathrm{w}}}{S}\right) \\
& J=\frac{q_{\mathrm{c}}}{1+v} \frac{\rho_{\mathrm{G}}^{2}}{\rho_{\mathrm{L}}} \sqrt{\frac{2 \sigma_{\mathrm{S} / \mathrm{G}}}{\pi m^{3}}} \exp \left(\frac{-\Delta G^{*}}{k T_{\mathrm{G}}}\right)
\end{aligned}
$$

where $\rho_{\mathrm{L}}$ is the density of the chemical droplet, $r^{*}$ is the critical radius, $v$ is the nonisothermal correction factor of Kantrowitz [16], $q_{\mathrm{c}}$ is the condensation coefficient, $m$ is the mass of a water vapour molecule, and $k$ is Boltzman's constant.

In the case of homogeneous nucleation, no impurities exist in the steam and therefore all impurity-related terms can be discarded. Taking the activity coefficient of pure water to be $a_{\mathrm{w}}=1$, homogeneous nucleation can be considered as a limiting case of the heterogeneous nucleation with the impurity fraction approaching zero.

It can be deduced from equations (1) and (2) that impurities in the steam promote the occurrence of the nucleation phenomena and decrease the subcooling demand because $a_{\mathrm{w}}$ is always smaller than 1.0 in the case of heterogeneous nucleation.

\subsection{Supersaturation and expansion rate}

Because the important focus in the present investigation lies in the metastable steam region immediately before nucleation, the nucleation position, and the nucleation phenomenon itself, we are not particularly interested in the following droplet growth process. The latter shall be approximated by considering the generated two-phase mixture to be in thermal equilibrium. This approximation is reasonable since a high number of very small droplets is formed and the fraction of secondary droplets is negligible in the first stages.

Neglecting the droplet growth process, however, brings up the necessity of a powerful criterion to determine the nucleation position as well as the associated nucleation rates and critical droplet sizes. This is done by application of a Wilson surface concept, derived in detail by Bohn et al. [15], which ends with the formulation of a necessary Wilson supersaturation for maximum nucleation described by:

$$
\ln S_{\mathrm{w}}=A\left(\frac{\dot{P}}{\sec ^{-1}}\right)^{B}\left[\frac{T_{\mathrm{c}}}{T_{\mathrm{G}}}-1\right]^{1.5}+\ln a_{\mathrm{w}}
$$

where $\dot{P}$ is the expansion rate, $(A=0.9816)$ and $(B=0.0595)$ are empirical parameters fitting experiments with expansion rates within the range of $2 \times 10^{1} \mathrm{sec}^{-1}$ to $2 \times 10^{5} \mathrm{sec}^{-1}$ and temperatures of the gaseous phase between $285 \mathrm{~K}$ and $400 \mathrm{~K}$, which is well suited for the steam turbine flow investigated.

Figure 3 shows the resulting Wilson surfaces for homogeneous and heterogeneous nucleation conditions, respectively. It can be seen that the impurity content ( $a_{\mathrm{w}}$ smaller than unity) shifts the surface towards lower necessary supersaturations.

\subsection{Governing equations, numerical procedure and grid}

The numerical scheme for the simulation of the fluid flow is based on an implicit finite volume method combined with a multi-block technique for structured grids. The physical domain is divided into separate blocks and the full, compressible, three-dimensional Navier-Stokes equations are solved in the fluid blocks. The governing equations for the conservative variables are developed in arbitrary, body-fitted coordinates $\xi, \eta$, $\zeta$ with the fluxes in normal directions to $\xi, \eta, \zeta=$ const.

The conservation equations are discretized implicitly first order in time using Newton's method [17]. Upwind discretization is used for the inviscid fluxes. A Godunov-type

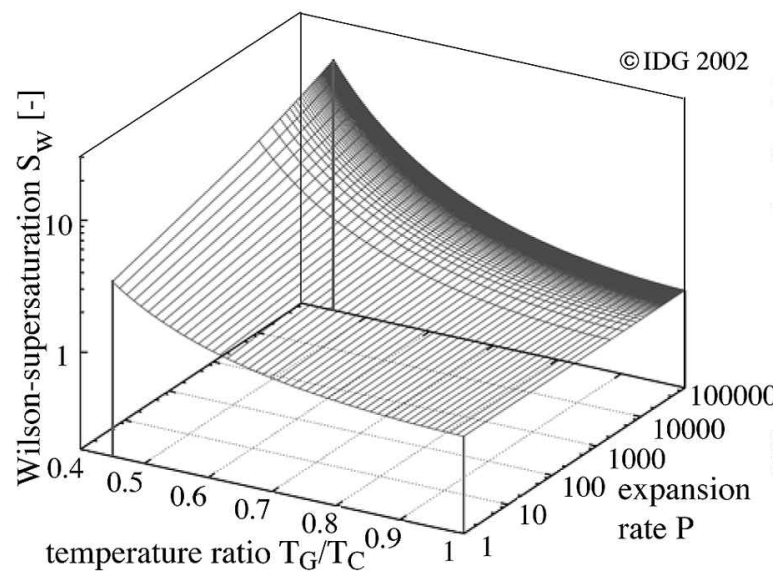

a) homogeneous nucleation

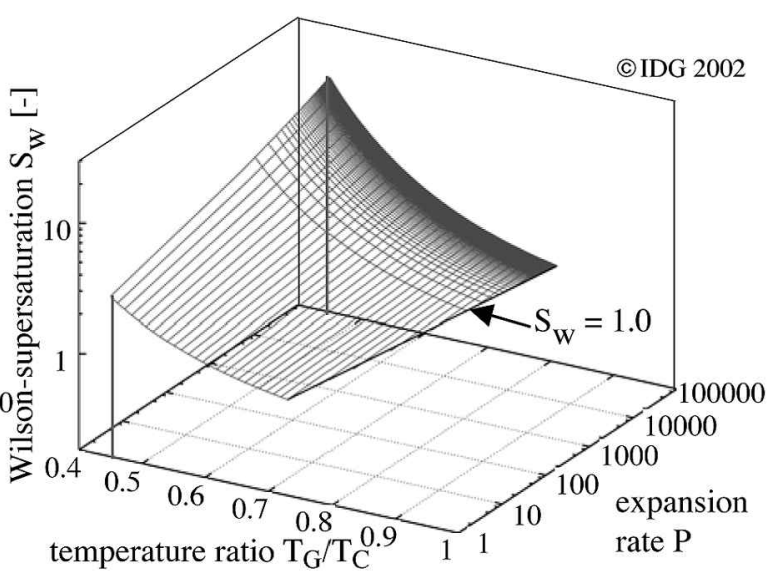

b) heterogeneous nucleation

Fig. 3 Wilson surfaces (necessary supersaturation) 


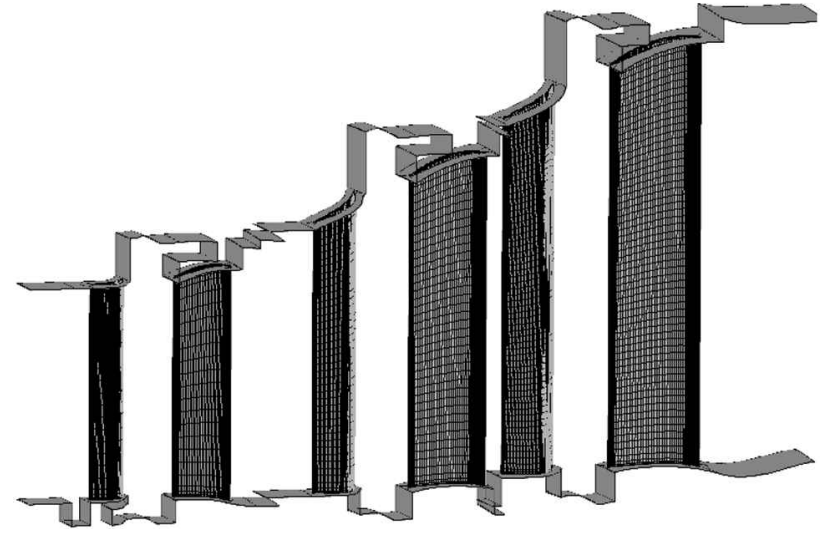

Fig. 4 Computational flow domain

flux differencing is employed for reducing numerical diffusion. In order to achieve third-order accuracy in space, van Leer's MUSCL-technique [18] is used. Since this Godunov flux is not sufficiently diffusive to guarantee numerical stability in regions with high gradients, it is combined with a hyperdiffusive modified Steger-Warming flux. The viscous fluxes are approximated using central differences. The resulting system of linear equations is solved by a Gauss-Seidel point iteration scheme allowing high vectorization on today's computers. The closure of the conservation equations is provided by the algebraic eddy-viscosity turbulence model by Baldwin and Lomax [19]. The performance of the code has been proven in a benchmark calculation [20] of a 1.5-stage cold air axial flow turbine [21].

Convergence is ensured by the two conventional necessary criteria, residuals of the conservative variables and the mass flux across the mixing planes. The irreversibly averaged isentropic parameter of expansion [22] is employed as the sufficient convergence criterion, since it also incorporates indirectly the dissipation and the corresponding temperature field development during the iterative calculation procedure.

The complete flow field is calculated under real metastable steam conditions. The temperature field and the transport properties are calculated by steam table subroutines according to the IAPWS Industrial Formulation 1997 [23]. According to the presented nucleation model, the nucleation position is determined. In a following step, the equilibrium wet-steam flow field with wetness-corrected pressure and temperature distributions is calculated by means of the conservation of the internal specific energy in each cell downstream of the nucleation. For a more detailed discussion of the real gas implementation for steam, see the work by Bohn et al. [24].

The droplet transport is considered by solving a firstorder convective/diffusive differential transport equation for the droplet numbers with the calculated single-phase flow field as input. The nucleation rate acts as source term on the right-hand side of the differential equation. Combining its result with the equilibrium wetness fraction, droplet diameter distributions can be derived.

Figure 4 gives an impression of the numerical grid employed to model the three axial front stages. In all, 42 blocks totalling approximately 2.4 Mio cells make up the computational domain. In order to incorporate the viscous loss mechanisms in the endwall regions, the open shroud cavities are taken into account. The parasitic labyrinth leakage flows through the finite radial clearances are not considered. "Mixing planes" are applied between stationary and rotating parts using a flux coupling method, yielding an excellent transport of conservative fluxes and fluid properties.

For this numerical study, the boundary conditions are chosen close to a corresponding testing condition with a final wetness of little more than 14 per cent behind the final stage (CS 62). At the inlet, swirl-free inflow is assumed with a total inlet pressure of about $2.7 \mathrm{bar}$ and a total inlet temperature of $405 \mathrm{~K}$. At the exit a constant static pressure of $0.55 \mathrm{bar}$ is prescribed. These values yield a reasonable representation of the real flow conditions.

\subsection{Numerical results}

Figure 5 shows the positions of the two different nucleation fronts. For both homogeneous (Fig. 5a) and heterogeneous

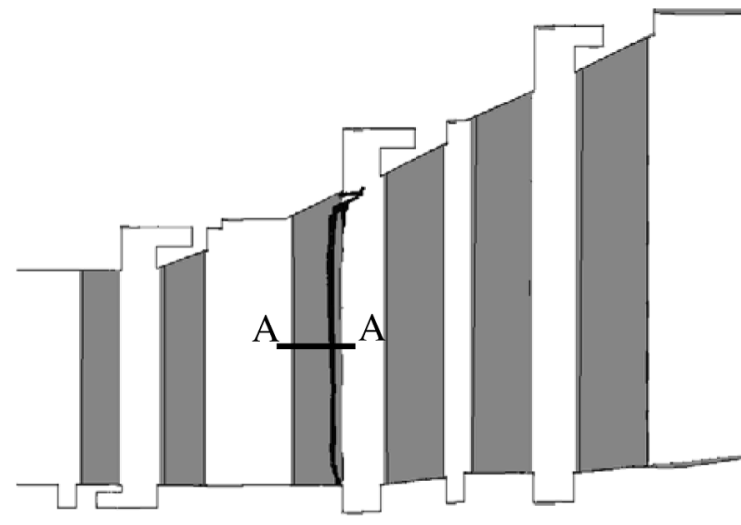

a) homogeneous nucleation

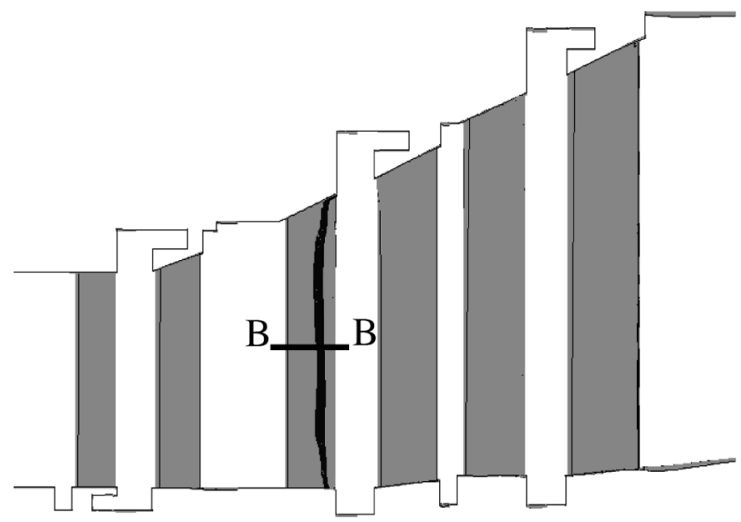

b) heterogeneous nucleation

Fig. 5 Nucleation front (meridional cut) 


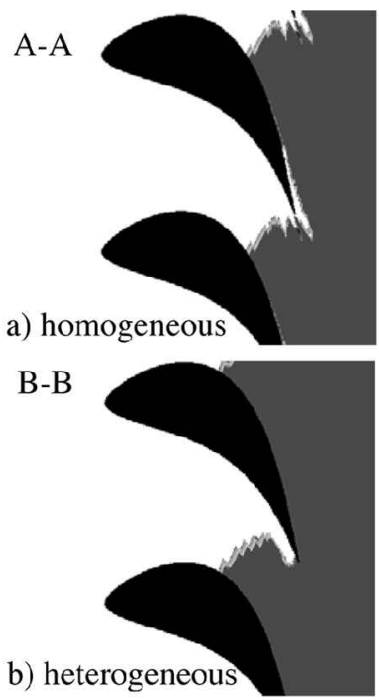

2nd stator c) expansion rate (2nd stage)

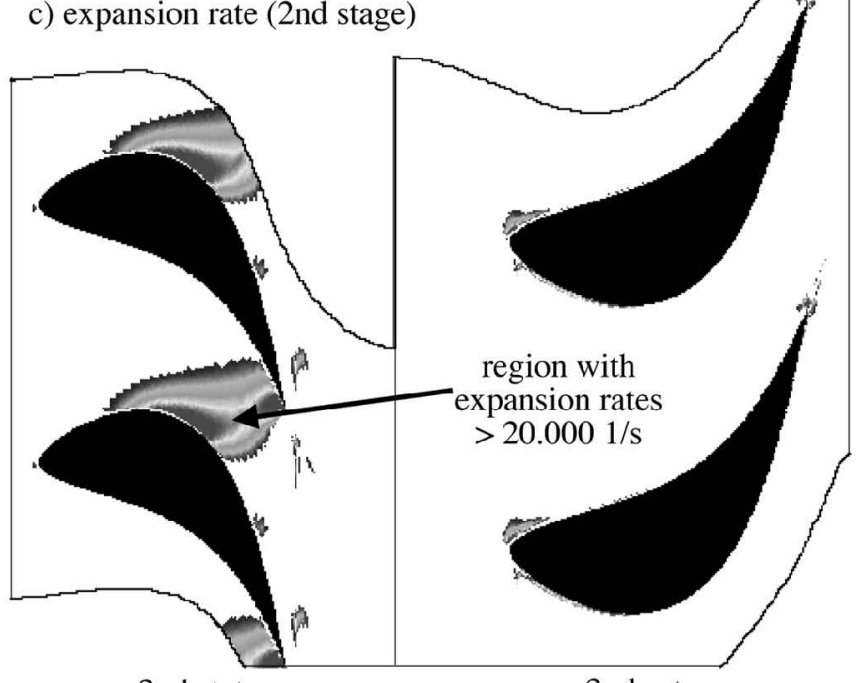

2nd stator

2 nd rotor

Fig. 6 (a), (b) Position of the nucleation front and (c) distribution of expansion rate at channel mid-height (second stage)

(Fig. 5b) nucleation, the position is located within the second stage's nozzle guide vane. As noted before, the impurities in the steam promote the nucleation to occur a little earlier. Owing to the dissipative viscous effects near the endwalls and inside the open shroud cavities the shape of the nucleation fronts is convex in the flow direction, as has been shown before, for example, by Kreitmeier et al. [25]. The convex shape is supposed to be even stronger, if the labyrinth leakage is incorporated.

Figure 6 shows some aspects of the nucleation position in a blade-to-blade cut at channel mid-height. Because the swirl and, correspondingly, the high velocity level is reduced inside the rotor, the highest expansion rates occur preferably in the nozzle guide vanes. In particular, near the throat, values greater than $200001 / \mathrm{s}$ are present. Up- and downstream of this region the expansion rate exhibits values of less than 10001/s. The heterogeneous nucleation front is moved in the upstream direction towards a region of higher expansion rates but lower subcooling and lower supersaturation, which, in terms of necessary Wilson supersaturation, counteract each other. In Fig. 7 the course of expansion rate and supersaturation is displayed along the dimensionless length of the nucleation front at channel mid-height.

In Fig. 8 the resulting nucleation rates along the dimensionless nucleation front length are shown. Clearly visible is the largely increased number of critical droplets. Responsible for this is the impurity content, incorporated in our model via the activity coefficient $a_{\mathrm{w}}$. As has been shown before for other steam flows $[\mathbf{1 5}, \mathbf{2 4}]$, besides being more numerous, the critical droplets are also smaller. Propagating the nucleated droplet population downstream to the third
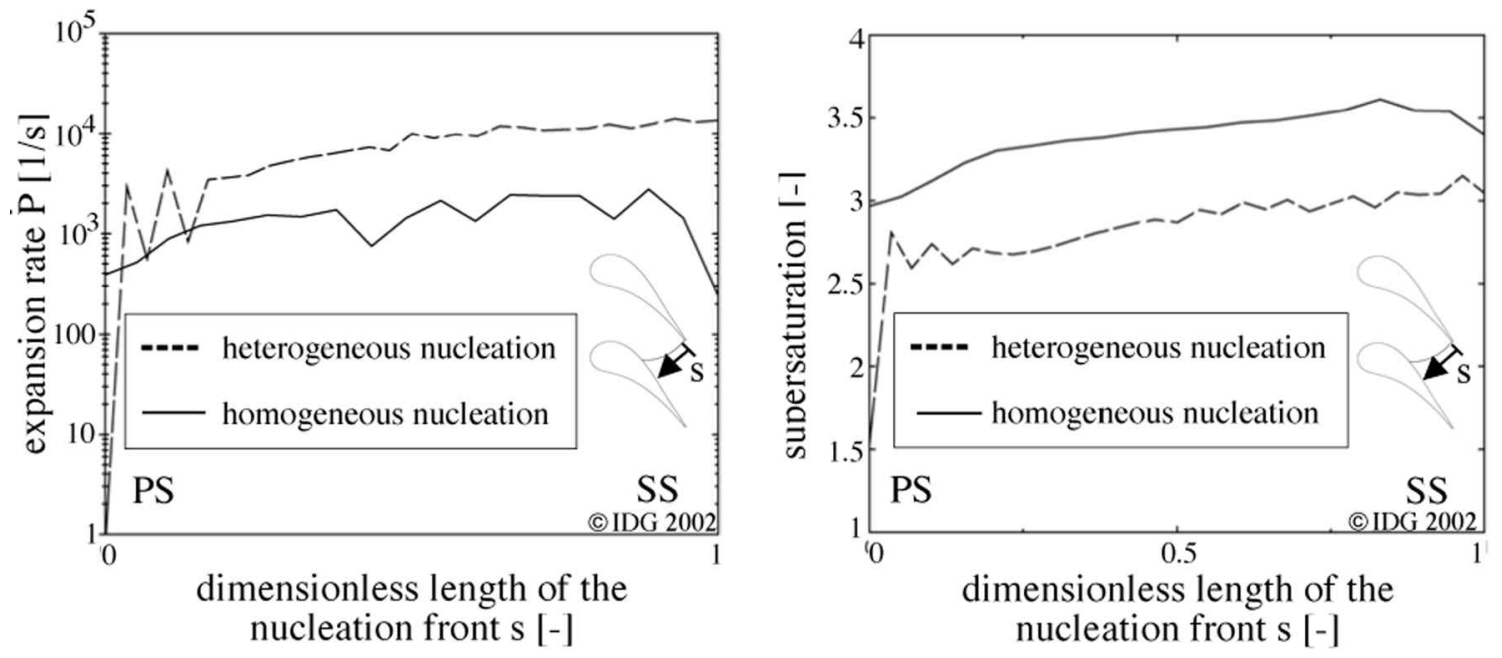

Fig. 7 Distribution of expansion rate and supersaturation along the nucleation front 


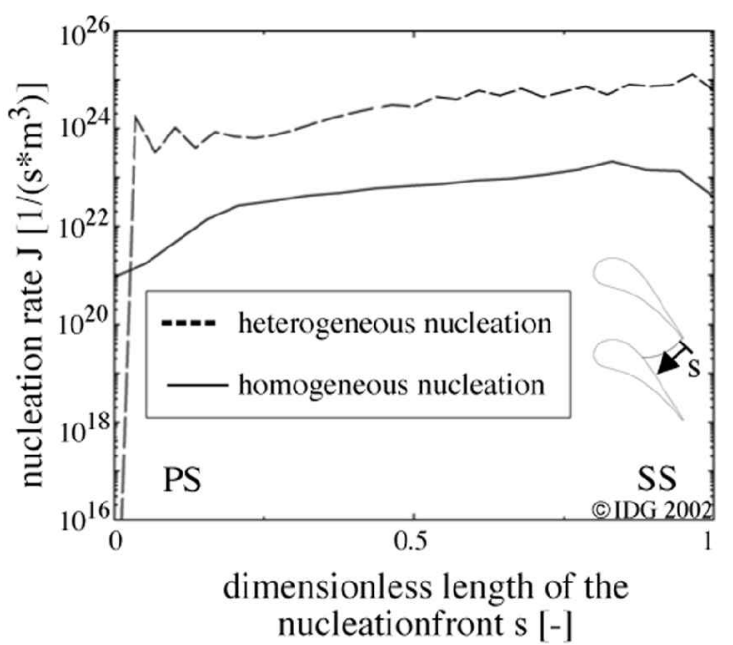

Fig. 8 Distribution of nucleation rate along the nucleation front

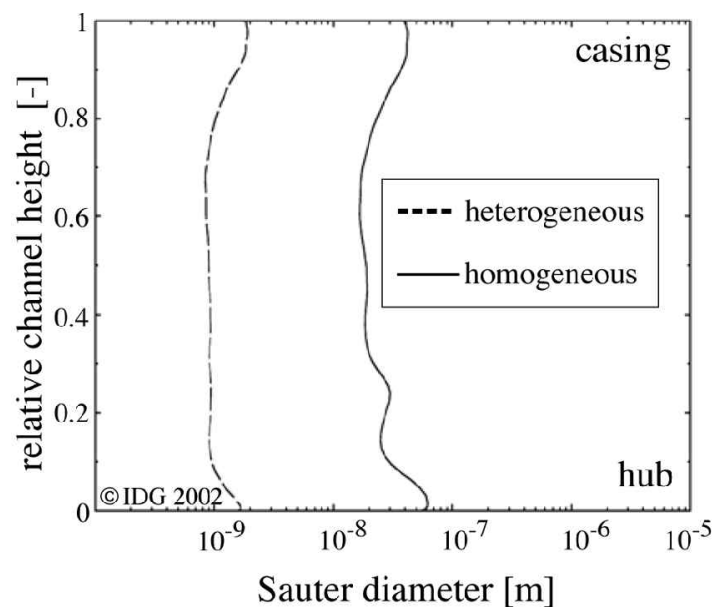

stage's exit plane (CS 42) results in the circumferentially averaged radial droplet Sauter diameter distributions and droplet number distributions shown in Fig. 9. The Sauter diameter is a droplet surface averaged droplet diameter incorporating information about the dispersion of the wetness. So we obtain the droplet density spectra depicted in Fig. 10. The spectra represent the droplet sizes across one pitch behind the third stage at about 50 per cent relative channel height. Looking at the values of the individual Sauter diameters it can be stated that in each of the two cases the liquid phase is nearly monodispersed. In particular, the homogeneously nucleated wet phase corresponds well to measured droplet sizes of similar experimental set-ups [25].

Besides the important but trivial conclusion that the increased nucleation rate in the case of impure steam results in smaller and more droplets in CS 42, it can be seen that the vast majority of droplets is found aside

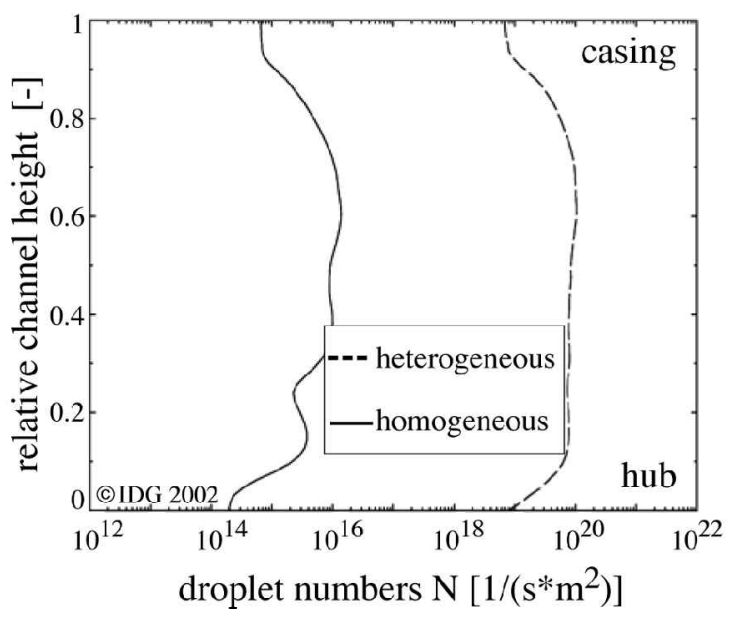

Fig. 9 Radial distributions of circumferentially averaged droplet size and droplet numbers in Control Surface 42 behind the third stage
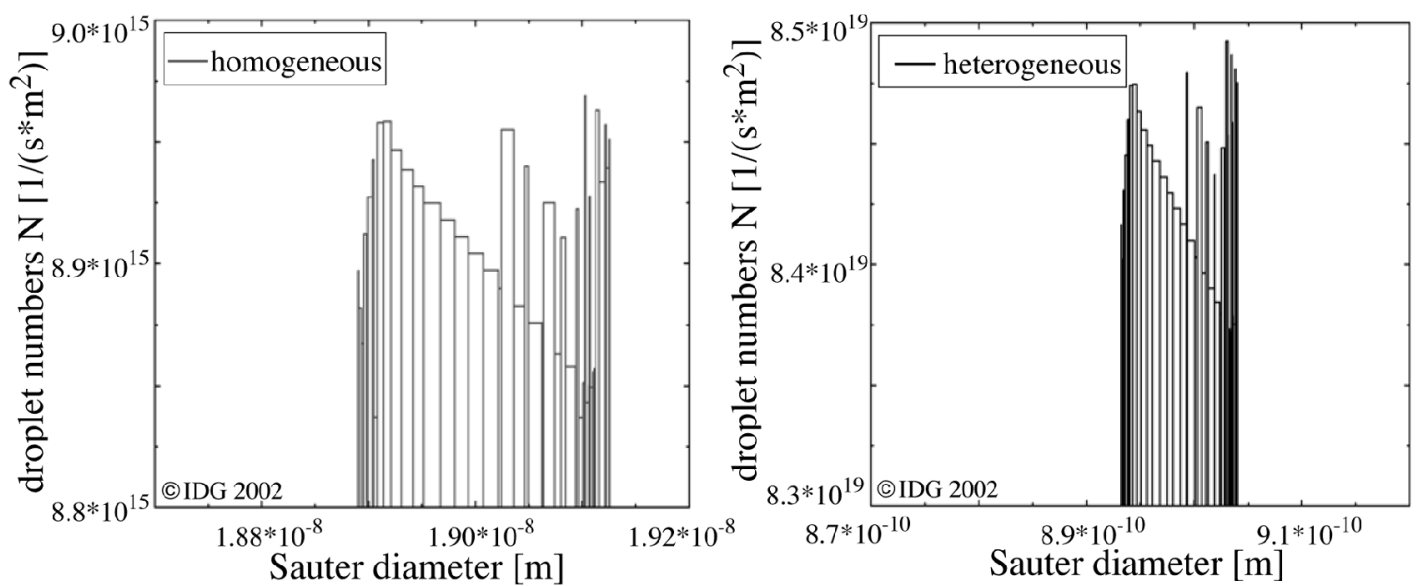

Fig. 10 Droplet density spectra for homogeneous and heterogeneous nucleated wet steam in Control Surface 42 behind the third stage (radial position: channel mid-height) 


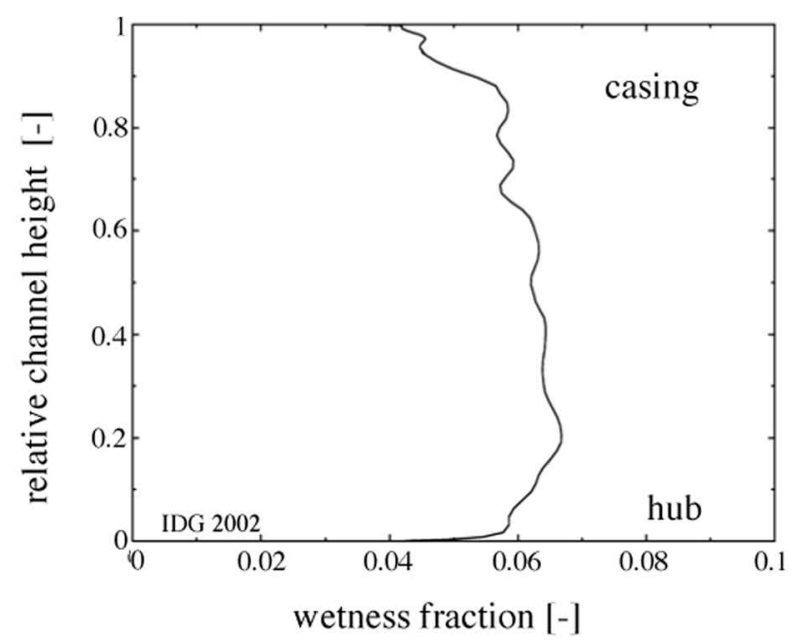

Fig. 11 Radial distribution of wetness fraction in Control Surface 42 behind the third stage

the endwall regions. This coincides with the radial distribution of wetness, shown in Fig. 11. Also here, the viscous effects due to endwall friction and vortex systems inside the shroud cavities are responsible for this characteristic shape.

\section{CONCLUSIONS}

In this paper a three-dimensional nucleating wet steam flow with homogeneous/heterogeneous nucleation in the three front stages of an industrial LP-steam turbine was investigated numerically. In order to take into account the additional viscous effects due to shrouded bladings, the open shroud cavities are modeled in detail. Droplet density spectra, radial droplet number, droplet diameter, and wetness fraction distributions at the exit of the third stage are calculated.

It is shown that impurities can cause nucleation to appear at lower supersaturations with higher nucleation rates compared to homogeneous nucleation of pure steam. In this way, thermodynamic and kinematic relaxation losses are reduced. Owing to the dissipative viscous effects near the endwalls, the nucleation fronts exhibit convex shapes. They are locally bound within the region of high expansion rates in the second stage's nozzle guide vane. For both heterogeneous and homogeneous nucleating flows, the wetness is highly dispersed with narrow droplet density spectra behind the three front stages.

\section{ACKNOWLEDGEMENTS}

The investigations were conducted as part of the joint research program "500 MW auf einer Welle (AG Turbo II)." The work presented was supported by the Bundesministerium für Wirtschaft (BMWI) under file number 0327061E. The authors gratefully acknowledge AG Turbo and ALSTOM Power for their support and permission to publish this paper. The responsibility for the content of this publication lies with the authors.

\section{REFERENCES}

1 Oswatitsch, K. Kondensationsstöße in Lavaldüsen. Z. Ver. deutscher Ing., 1942, 86, 402.

2 Frenkel, J. Kinetic Theory of Liquids, 1946 Oxford University Press, Oxford.

3 Dunning, W. J. General and theoretical introduction. In Nucleation, 1969 (Marcel Dekker, New York).

4 McDonald, J. E. Homogeneous nucleation of vapour condensation. Am. J. Phys., 1962, 30, 870.

5 Bohn, D. and Kerpicci, H. Nucleation of droplets and homogeneous condensation in a nozzle guide vane of a LP-Steam turbine. First International Conference on Engineering Thermophysics, 1999, Beijing, China.

6 Young, J. B. Two dimensional, nonequilibrium wet-steam calculations for nozzle and turbine cascades. J. Turbomachinery, 1992, 114, 569-579.

7 Köhler, H. The nucleus in and the growth of hygroscopic droplets. Trans Farad. Soc., 1936, 32, 1152-1161.

8 Fletcher, N. H. The Physics of Rainclouds, 1962 (Cambridge University Press, Cambridge).

9 Schnerr, G. H. Phase transition in fluid mechanics. Proc. 2nd Int. Symp. on Exp. and Computational Aerothermodynamics of Internal Flows, 1993, 1, 53-75.

10 Stastny, M. and Sejna, M. Numerical analysis of heterohomogeneous condensation of the steam flowing in turbine cascade. IMechE, C557/082/99, 1999, 815-826.

11 Gorbunov, B. Free energy of embryo formation for heterogeneous multicomponent nucleation. J. Chem. Phys., 1999, 110(20), 10035-10045.

12 Bohn, D. and Ren, J. Numerical research on the heterogeneous condensation in a nozzle guide vane of a LP-steam turbine. Proc. 2nd International Conference on Fluid Machinery and Fluid Engineering, 2000, Beijing, China, 314-321.

13 Kreitmeier, F., Juvet, P. and Weiss, P. Proactive product and process qualification in steam turbine development. $\mathrm{ABB}$ Review 4/1998, 1998, pp. 37-47.

14 Bohn, D. and Ren, J. Salt solution and radius dependence on the surface tension in the heterogeneous nucleation in steam turbines. Chinese Engineering Thermophysics Conference, October 11-17, 2001, Qingdao, China.

15 Bohn, D., Kerpicci, H., Ren, J. and Sürken, N. Homogeneous and heterogeneous nucleation and wet-steam flow in a nozzle and an LP-turbine cascade. Proc. 9th Int. Symp. on the Transport Phenomena and Dynamics of Rotating Machinery, 2002. Honolulu, Hawaii.

16 Kantrowitz, A. Nucleation in very rapid vapour expansions. J. Chem. Phys., 1951, 19, 1097-1100.

17 Eberle, A., Schmatz, M. A. and Bissinger, N. Generalized flux vectors for hypersonic shock-capturing. AIAA -paper 90-0390, 1990.

18 Anderson, W. K., Thomas, J. L. and van Leer, B. A comparison of finite volume flux vector splittings for the Euler equations. AIAA-paper 85-0122, 1985. 
19 Baldwin, B. S. and Lomax, H. Thin layer approximation and algebraic model for separated turbulent flows. AIAA-paper 78-257, 1978.

20 Emunds, R., Jennions, I. K., Gier, J. and Bohn, D. The computation of adjacent blade-row effects in a 1.5 stage axial flow turbine. ASME-paper 97-GT81, 1997.

21 Walraevens, R. E. and Gallus, H. E. Testcase 6-1.5 stage axial flow turbine. ERCOFTAC Testcase 6. IST-Report, Aachen University, 1997.

22 Bohn, D., Funke, H., Sürken, N. and Kreitmeier, F. Numerical and experimental investigations on endwall contouring in a four-stage turbine. ASME-paper 2001-GT481, 2001.
23 Wagner, W., Cooper, J. R., Dittmann, A., Kijima, J., Kretzschmar, H.-J., Kruse, A., Mares, R., Oguchi, K., Sato, H., Stöcker, I., Sifner, O., Takishi, I., Trübenbach, J. and Willkommen, Th. (2000). The IAPWS industrial formulation 1997 for the thermodynamic properties of water and steam. Trans. ASME, 2000, 122, 150-182.

24 Bohn, D., Kerpicci, H., Ren, J. and Sürken, N. Homogeneous and heterogeneous nucleation in a nozzle guide vane of a LPsteam turbine. Proc. 4th European Conference on Turbomachinery, 2001, Florence, Italy.

25 Kreitmeier, F., Schlachter, W. and Smutny, J. Strömungsuntersuchungen in einer Niederdruck-Modellturbine zur Bestimmung der Nässeverluste. VDI-Berichte Nr. 361, 1980, 201-211. 